\title{
Reconstruction of the solidification front inside an icing drop
}

\author{
M.STITI*1, G.CASTANET ${ }^{1}$, A.LABERGUE ${ }^{1}$, F.LEMOINE ${ }^{1}$ \\ ${ }^{1}$ Université de Lorraine, CNRS, LEMTA, F-54000 Nancy, France \\ ${ }^{*}$ Corresponding author email: mehdi.stiti@outlook.fr
}

\begin{abstract}
We present here a study of the solidification process of water droplets and two subcooled substrates having highly different thermal properties: duralumin (high heat conductivity) and N-BK7 glass (low heat conductivity). An experimental technique based on laser-induced fluorescence is developed to reconstruct the evolution of the ice front within the freezing droplets. Comparisons between the two substrates reveals how the freezing dynamics can have a significant effect on the shape of the freezing front. Early in the freezing process, the ice front displays a spherical shape for both substrates. However, the curvature of the ice front and the angle with the free liquid surface are dependent on the freezing rate and the conjugate heat transfer within the substrate material. In the condition of the experiments (droplets injected at an initial temperature of $18^{\circ} \mathrm{C}$ and a wall temperature of $-10^{\circ} \mathrm{C}$ ), the formation of a pointy tip at the end of the freezing process is only observed in the case of N-BK7 glass. The contact angle at the solid/liquid/air tri-junction point allows explaining the formation of this pointy tip. A simplified 2D axisymmetric model is compared to the experimental results.
\end{abstract}

\section{Keywords}

Laser Induced fluorescence, Droplet freezing, Solidification

\section{Introduction}

During their flight, aircraft may encounter icing conditions that can create serious hazards. Icing conditions are characterized by the presence of supercooled water responsible for the accumulation of ice on aircraft surfaces, thus reducing flight performance (Cebeci, T; Kafyeke 2003). It is essential to understand the solidification mechanisms of a droplet (Hindmarsh et al. 2005; Marín et al. 2014; Schremb et al. 2017; Thiévenaz et al. 2019), to design surfaces that can reduce the effects of icing.

\section{Experimental setup}

The experimental setup is depicted in Figure 1 . To generate the water droplet, a $400 \mu \mathrm{m}$ diameter needle is connected to a micro-pump. The droplet is detached from the needle when the droplet diameter is equal to $2.6 \mathrm{~mm}$. The impact velocity $(u)$ of the free-falling droplet increases with the distance $h$ between the needle and the solid substrate $(u=\sqrt{2 g h})$. Hence, changing the distance $h$ allows controlling the Weber number (defined by $W e=\rho u^{2} d / \sigma$ where $\mathrm{d}$ is the drop diameter, $\rho$ the liquid density and $\sigma$ the surface tension) and thus the spreading of the drop onto the solid surface. In this study, an impact velocity $u=1.35 \mathrm{~m} / \mathrm{s}$, corresponding to $W e=65$, is considered. The temperature of the solid surface is controlled by means of a heat exchanger. The solid substrate onto which the droplet impact takes place is inserted directly into a hole drilled on the surface of the heat exchanger. In this study, the focus will be placed on two kinds of materials: duralumin and optical glass (N-BK7) which have very different thermal properties (Table 1). Both substrates have a thickness of $5 \mathrm{~mm}$. A thermocouple allows controlling the temperature of the substrate. 


\begin{tabular}{|c|c|c|c|c|c|}
\cline { 2 - 6 } \multicolumn{1}{c|}{} & $\begin{array}{c}\rho \\
{\left[\mathrm{kg} \cdot \mathrm{m}^{-3}\right]}\end{array}$ & $\begin{array}{c}\mathrm{Cp} \\
{\left[\mathrm{W} \cdot \mathrm{m}^{-1} \cdot \mathrm{K}^{-1}\right]}\end{array}$ & $\begin{array}{c}D \\
{\left[\mathrm{~J} \cdot \mathrm{kg}^{-1} \cdot \mathrm{K}^{-1}\right]}\end{array}$ & $\begin{array}{c}e \\
{\left[\mathrm{~m}^{2} \cdot \mathrm{s}^{-1}\right]}\end{array}$ & {$\left[\mathrm{J} \cdot \mathrm{K}^{-1} \mathrm{~m}^{-2} \mathrm{~s}^{-\frac{1}{2}}\right]$} \\
\hline Duralumin & 2700 & 145 & 897 & $0.6 \times 10^{-5}$ & $1.9 \times 10^{4}$ \\
\hline N-BK7 & 2510 & 1.114 & 858 & $5.2 \times 10^{-7}$ & $1.6 \times 10^{3}$ \\
\hline \hline Water $\left(20^{\circ} \mathrm{C}\right)$ & 1000 & 0.6 & 4180 & $1.5 \times 10^{-7}$ & $1.6 \times 10^{3}$ \\
\hline Ice $\left(0^{\circ} \mathrm{C}\right)$ & 917 & 2.2 & 2050 & $1.2 \times 10^{-6}$ & $2 \times 10^{3}$ \\
\hline
\end{tabular}

Table 1 Thermophysical properties of the substrates and water.

The experimental system (droplet and substrate) operates in air at room temperature without humidity control. Because of this, a thin layer of ice quickly develops on the substrate before each impact experiment. The presence of this frost layer allows obtaining a great reproducibility of the freezing phenomenon. The freezing starts immediately after the droplet impinges onto the solid surface. Thus, there is no supercooling of the liquid water, as there is no time delay for the liquid to be cooled below the melting point before the icing. No recalescence events can be observed in the experiments.

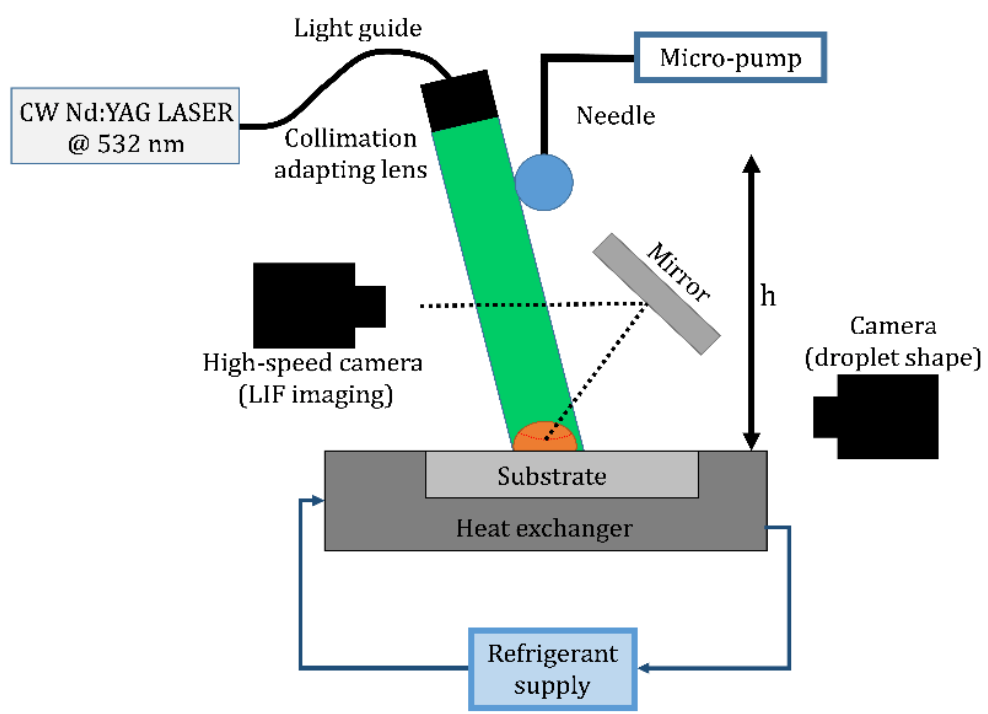

Figure 1 Experimental setup.

\section{Observation of the droplet impact and freezing}

Shadow imaging was used to visualize the evolution of the droplet during the impact and the freezing process. As an example, Figure 2 shows the droplet evolution for two similar impinging conditions, and two substrates made of different material: duralumin and N-BK7 glass. For these two impacts, the initial wall temperature was $T_{0}=-10^{\circ} \mathrm{C}$ and the impact velocity was $u=1.35 \mathrm{~m} / \mathrm{s}$. During the initial stage of the impingement, the same spreading evolution is observed for both materials due to the initial presence of frost. At the end of spreading process (roughly about $5 \mathrm{~ms}$ ), the spreading diameter is approximately 2.5 times the initial droplet diameter. After having reached the maximum spreading, no noticeable recoiling of the droplet occurs. Due to the initial frost layer, freezing can start very early near the triple contact line as the spreading of the liquid decelerates. This subsequently blocks any motion of the triple line (Schremb et al. 2018). The freezing process takes a much longer time than the droplet spreading. The duration of the drop freezing is mainly a function of the thermal properties of the solid wall. Experimentally, the measured freezing time is $22 \mathrm{~s}$ for N-BK7 and 
$2.2 \mathrm{~s}$ for duralumin, which has a much larger heat conductivity. A noticeable difference between the experiments carried out on the two substrates, is the formation of a pointy tip at the end of the solidification phase in the case of N-BK7 (Figure 2). This tip is absent in the case of the duralumin substrate.

a)

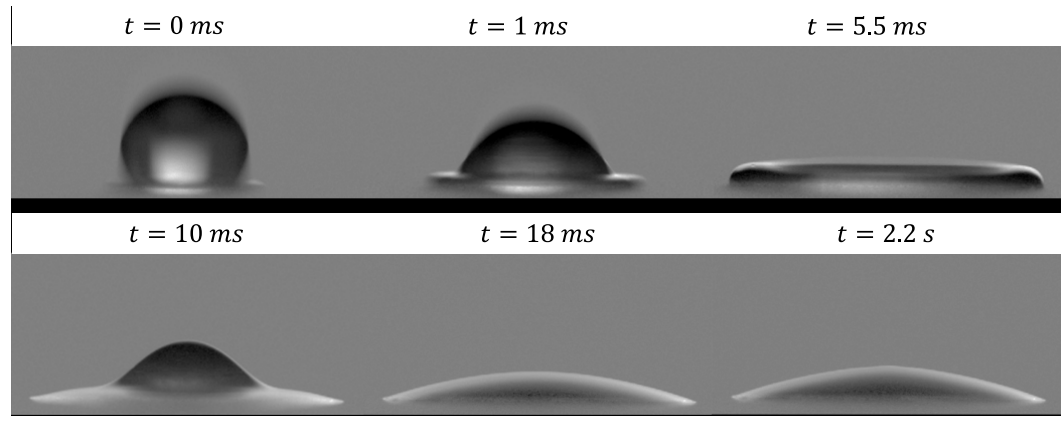

b)

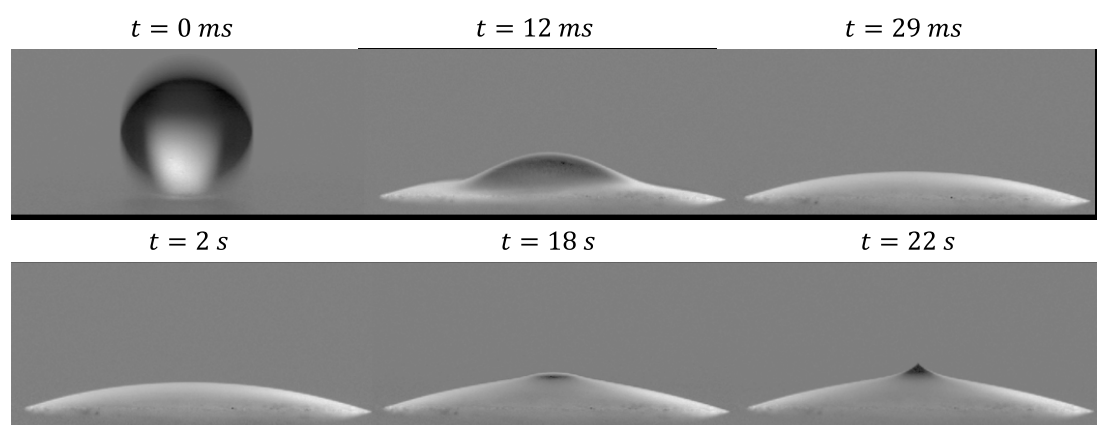

Figure 2 Images of the freezing of a droplet impinging a subcooled $\left(\mathrm{T}=-10^{\circ} \mathrm{C}\right)$ surface at $W e=65$. (a) Duralumin and (b) N-BK7 glass.

\section{Laser-induced fluorescence signal within a freezing droplet}

To obtain quantitative data on the icing, the ice front is reconstructed based on Laser Induced Fluorescence (LIF) signal. Basically, the method consists in dissolving an organic fluorescent dye in water prior to the droplet generation. A laser excitation tuned on the absorption spectrum of the dye molecule, is then used to induce the fluorescence during the icing of the droplet. The resulting fluorescence signal is observed by a camera looking at the droplet from above. Then, the fluorescence images are analysed to determine the $2 \mathrm{D}$ liquid thickness and thus the position of the ice front. In practice, a high-speed camera (phantom V710) is required given the short duration of the icing phenomenon. A tilted mirror is placed at the top of the droplet in order to obtain top view images of the droplet (Figure 1). A CW frequency-doubled Nd-YAG laser (@532 nm; P=2.5W) is used to induce the fluorescence. The laser beam is transmitted by a light guide consisting of a bundle of optical fibres. Rhodamine 6G (R6G) has been selected as fluorescent marker since his signal in the solid phase is negligible in front of the one in the liquid phase. A long-pass edge filter, transmitting longer wavelengths than 542 $\mathrm{nm}$, is mounted in front of the camera lens to eliminate the contribution of laser scattering and to detect only the contribution of the LIF signal. It can be shown that the fluorescence signal $I_{f}$ can be linked to the liquid thickness. By taking a reference $I_{f, r e f}$ where the liquid thickness is $Z_{L, r e f}$, the liquid thickness can be evaluated at any time during the freezing as followed:

$$
Z_{L}(x, y)=Z_{L, r e f}(x, y) \cdot \frac{I_{f}}{I_{f, r e f}} .
$$

Hence, it is possible to determine the liquid thickness $Z_{L}$ by using a reference image taken under the same spatial distribution of laser excitation $I_{0}(x, y)$. 
The main steps of the image post processing are:

Step 1: The raw fluorescence image is subtracted from the background noise. Step 2: An image of the laser intensity distribution can be obtained by positioning a thin fluorescent PMMA slab of uniform thickness at the same location as the droplet, prior to the measurement. The non-uniformity of the laser illumination is corrected by dividing the droplet image by the reference image from the plate. Step 3: The radial distribution of $I_{\text {norm }}$ is extracted from the previous image. An axysimetric averaging is performed, which helps in improving the SNR except near the droplet

centre.

Step 4: A geometrical scaling is required as $I_{\text {norm }}$ only provides a relative measurement of the liquid thickness. This scaling factor, denoted by $S_{F}$, can be determined from the initial droplet volume $\left(V_{0}\right)$. The value of $S F$ can be determined as follows:

$$
S_{F}=\frac{\int_{0}^{+\infty} 2 \pi r I_{f}(r) d r}{V_{0}}
$$

\section{Reconstruction of the ice front}

The previously described method provides only the thickness of the liquid top layer within the droplet. As an approximation to determine the position $Z_{I}$ of the ice front, the volume expansion due to the freezing can be neglected. Knowing the droplet profile $Z_{d}$ at $t=t_{0}$ ( $t_{0}$ is taken just after the dumping of the surface oscillations, i.e. just after $20 \mathrm{~ms}$ ), the height $Z_{I}$ of the ice front can be evaluated as follows:

$$
Z_{I}(R, t)=Z_{d}\left(R, t_{0}\right)-Z_{L}(R, t)
$$

Since the icing did not have time to propagate significantly during the impact process (which lasts only a few tens of $\mathrm{ms}), Z_{d}\left(R, t_{0}\right)$ can be deduced from the fluorescence signal.

In order to account for volume expansion in the reconstruction of the ice front, an additional camera is required. This allows obtaining sideview images of the droplet from which the actual shape and position of the droplet surface can be inferred. Denoting $Z_{d}$ the height of the droplet surface on the sideview images, the position of the ice front $Z_{I}$ can be determined as follows:

$$
Z_{I}(R, t)=Z_{d}(R, t)-Z_{L}(R, t)
$$

Equation (4) is similar to Eq.(3) except that $Z_{d}(R, t)$ is the height of the free surface at the time corresponding to the LIF image.

\section{Icing of a droplet deposited on the duralumin substrate}

In the following development, point $A$ will refer to the position of the ice front at the droplet center, and point $B$ at the tri-junction between the ice front, the liquid phase and the air. Figure 3(A) presents the time evolution of $Z_{B}$. The analysis of the experimental results shows that $Z_{B}$ increases following approximately as a square root of the time, which is congruent to results concerning the freezing of sessile droplets in the literature (Zhang et al. 2019). As presented in Figure 3(A), the 1D and 2D-axi simulations (presented in (Stiti et al. 2020)) are able to reproduce quite well this temporal evolution of $Z_{B}$. However, the motion of $Z_{B}$ in the simulation is slower than observed experimentally. The difference can be attributed to the volume expansion due to the freezing, which adds an additional velocity to the ice front. The best fit of the experimental data is $D_{\text {eff,exp }}=1.08 \times 10^{-7} \mathrm{~m}^{2} \cdot \mathrm{s}^{-1}$ while $D_{\text {eff,m }}$ is about $8 \times 10^{-8} \mathrm{~m}^{2} . \mathrm{s}^{-1}$ for the semi-infinite $1 \mathrm{D}$ without volume expansion. Figure $3(\mathrm{~B})$ also presents the evolution of the radial position $R_{B}$ of point $\mathrm{B}$. Considering that the shape 
of the deposited droplet can be assimilated to a spherical cap of radius $R, R_{B}$ is expected to follow a $R^{2}$-law (Marín et al. 2014):

$$
R_{B}=\sqrt{R^{2}-\left(Z_{B}-Z_{C}\right)^{2}}
$$

where $Z_{c}$ is the vertical position of the center of the spherical cap at the droplet free surface. Replacing $Z_{B}$ by $\sqrt{D_{\text {eff }} . t}$ yields:

$$
R_{B}^{2}-R^{2}=\left(\sqrt{D_{e f f} t}-Z_{c}\right)^{2} .
$$

The temporal evolution of $R_{B}$ predicted by Eq.(6) is plotted in Figure $3(\mathrm{~B})$ using $D_{\text {eff }}=$ $1.08 \times 10^{-7} \mathrm{~m}^{2} / \mathrm{s}$, which is the value obtained experimentally for $Z_{B}$.

A)

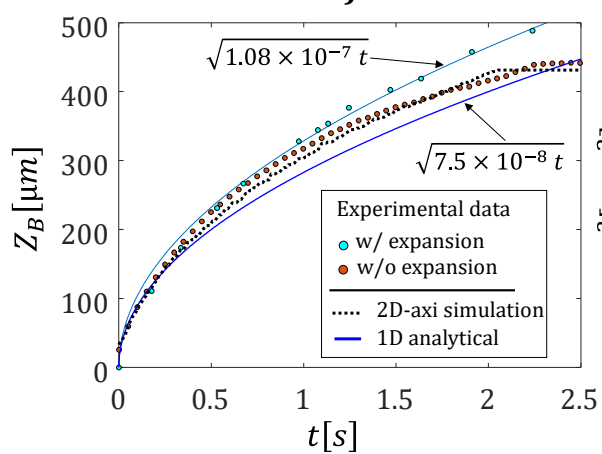

B)

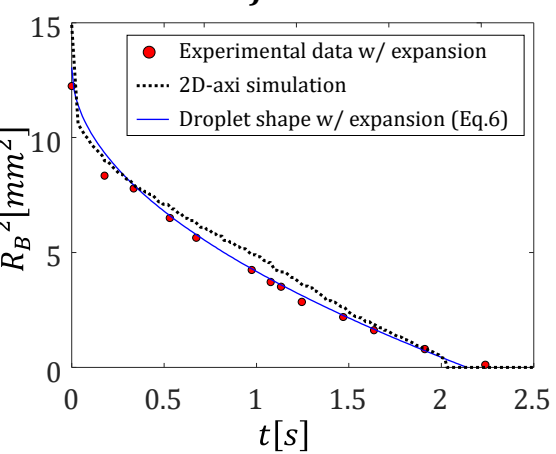

Figure 3 Evolution of the ice thickness $A$ ) and radial position $B$ ) of the ice front at the air/liquid/solid tri-junction (point $B$ ) in function of the time for $T_{0}=-10^{\circ} \mathrm{C}$ and $T_{\infty}=18^{\circ} \mathrm{C}$.

Figure 4 shows the time evolution of the ice front at point $\mathrm{A}$ (on the droplet axis at $R=$ 0 ) for the same icing conditions as in Figure 3 . The analysis of the experimental data reveals that $Z_{A}$ evolves as $t^{0.75}$. The 2D-axi simulation is able to reproduce this temporal evolution of $Z_{A}$ quite well, but in the very last instants of the freezing, typically for $t>2.1 \mathrm{~ms}$, a sudden acceleration of the ice front is observed. The volume expansion inherent to the freezing is certainly responsible for it since this acceleration is not captured by the 2D-axi simulation. If the ice front is reconstructed experimentally assuming no volume expansion like in the simulations, it is worth noting that the agreement between the experimental data and the simulation is a bit improved.

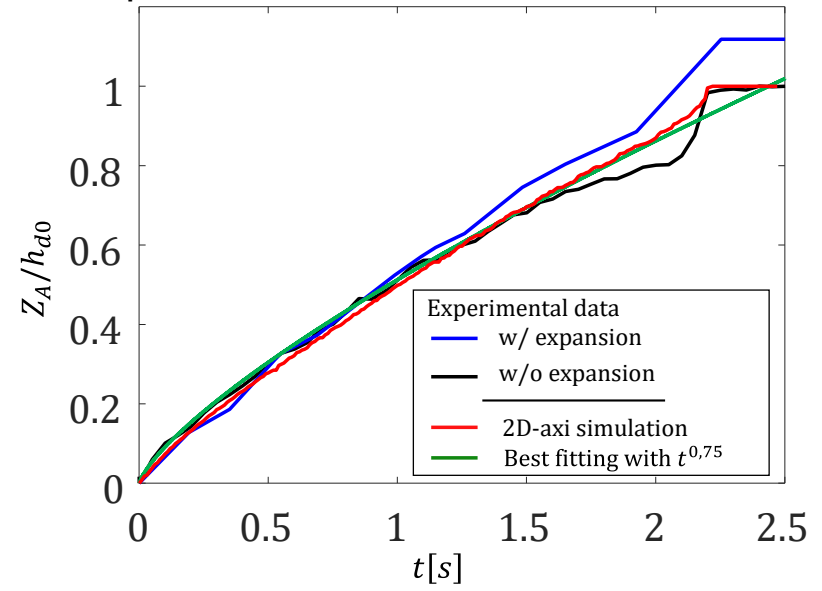

Figure 4 Evolution of the ice thickness at the droplet center $R=0$ (point $\mathrm{A}$ ). $h_{d 0}$ is the droplet height before freezing. Same freezing conditions as in Figure 3. 


\section{Comparisons between N-BK7 and duralumin substrate}

Figure 5 present the comparisons between the solidification front obtain numerically and experimentally in the case of a duralumin (Figure $5 \mathrm{~A}$ ) and N-BK7 (Figure $5 \mathrm{~B}$ ) substrate. Two main differences between the duralumin and N-BK7 substrate can be pointed out: In the case of a N-BK7 substrate, the freezing dynamics is much slower, and the ice front forms a much deeper cavity. These noticeable differences can be explained by the heat transfer between the solid wall and the droplet. Immediately after the droplet deposition, the temperature at the interface between the droplet and the wall reaches the thermal contact temperature $T_{c}$ defined by:

$$
T_{c}=\frac{T_{0} e_{s}+T_{\infty} e_{L}}{e_{L}+e_{s}}
$$

where $e_{L}$ and $e_{S}$ are the thermal effusivity of the liquid water and the solid. The application of Equation.(7) provides a contact temperature of $7^{\circ} \mathrm{C}$ in the case if the N-BK7 substrate and a temperature of $-8^{\circ} \mathrm{C}$ in the case of Duralumin surface. This difference allow the explanation of the delay of the freezing in the center in the case of the N-BK7 substrate.

A)

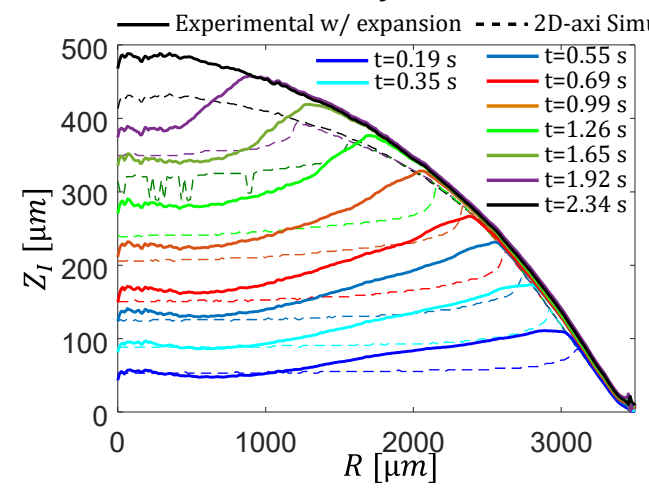

B)

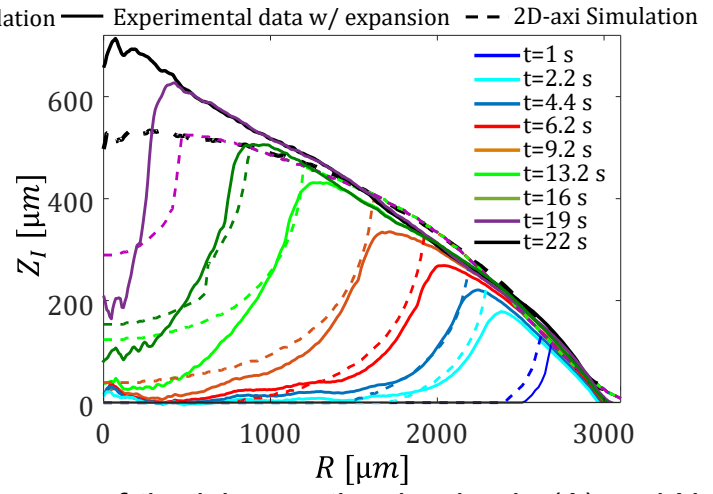

Figure 5 Evolution of the ice front profile in the case of the icing on the duralumin $(A)$ and N-BK7 (B) substrate in the same conditions as in Figure 3.

\section{Formation of the pointy tip}
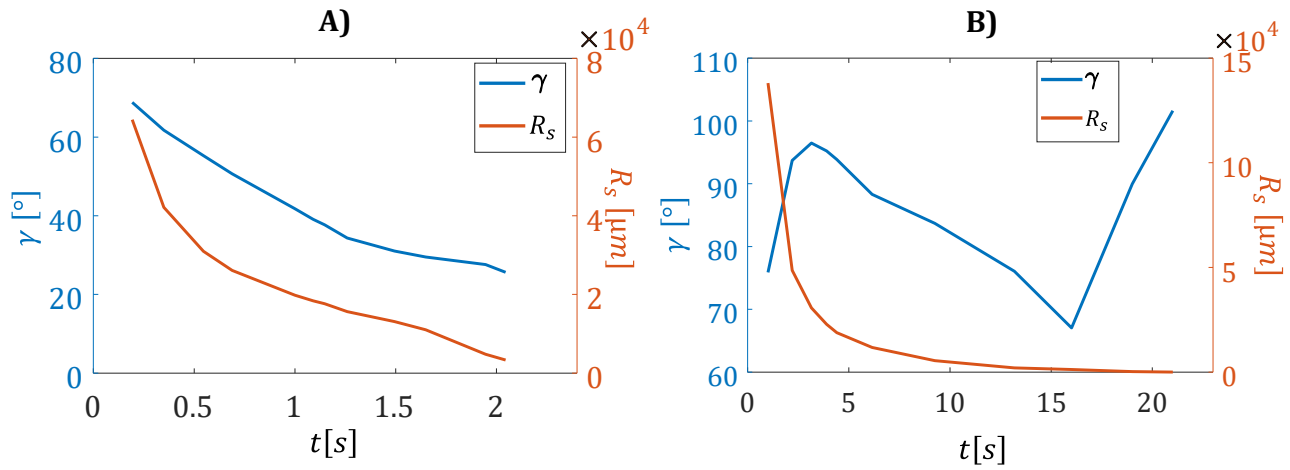

Figure 6 Evolution of the radius of curvature $R_{S}$ and the angle $\gamma$ for (A) duralumin and (B) N-BK7 substrates, cooled at $-10^{\circ} \mathrm{C}$.

The tip formation can be described taking into account the spherical shape of the ice front (Marín et al. 2014). Denoting $V_{L}$ and $V_{I}$ respectively the liquid and ice volume and $\psi$ the density ratio between liquid water and ice, the mass conservation yields:

$$
\frac{d}{d z}\left(V_{L}+\psi V_{I}\right)=0
$$

The liquid volume $V_{L}$ can be decomposed into a spherical cap of angle $\theta$ and a downward volume $V_{d}$ : 


$$
V_{L}=R^{3} f(\theta)+V_{d}
$$

The ice volume $V_{I}$ is a section of a spherical cap between $z=0$ and $Z_{B}$, from which the volume $V_{d}$ must be subtracted:

$$
V_{I}=\int_{0}^{z_{B}} \pi R\left(z^{\prime}\right)^{2} d z^{\prime}-V_{d}
$$

where $R(z)$ and $\theta(z)$ are respectively the local radius and angle of the frozen droplet. From geometrical consideration, regarding the spherical cap, the function $f(\theta)$ can be expressed as:

$$
f(\theta)=\frac{\pi}{3}\left(\frac{2-3 \cos (\theta)+\cos ^{3}(\theta)}{\sin ^{3}(\theta)}\right)
$$

Assuming that the ice front takes a spherical shape and denoting $\phi=\gamma-\theta$ :

$$
V_{d}=r^{3} f(\gamma-\theta)
$$

Using $d r / d z=\tan (\theta)$, the following relationship between $\theta$ and $\gamma$ can be deduced:

$$
f(\gamma-\theta)+f(\theta)=\psi\left[f(\gamma-\theta)+\frac{\pi}{3} \tan (\theta)\right] .
$$

Assuming that the pointy tip is a cone of angle $\alpha$ the previous equation can be used to determine the cone angle $\alpha=\pi-2 \theta$ of the pointy tip at the end of the freezing process (Marín et al. 2014; Zhang et al. 2019). However, an additional condition at the solid/liquid/air tri-junction (point $B$ ) is required for closing the problem. Recently, observations by Marín et al. (Marín et al. 2014) suggested that the conical tip angle $\alpha$ is independent of the substrate temperature and of the wetting angle. For water droplets, they measured a value of $\alpha$ equal to about $140^{\circ}$, whatever the freezing conditions. Using Eq. (13), this corresponds to $\gamma=$ $72^{\circ}, \gamma$ being the angle between the liquid interface and the ice front.

Error! Reference source not found. presents current experimental results for the contact angle $\gamma$ at the tri-junction (point $\mathrm{B}$ ) as well as the radius of curvature $R_{S}$ of the ice front. For both substrates, freezing dynamics induces a significant change in these two parameters. A decrease in $\gamma$ and $R_{S}$ can be observed during the first stage of the freezing for both duralumin and N-BK7 substrates. In the case of duralumin, $\gamma$ reaches an end value of about $27^{\circ}$. Based on Eq.(13), this yields a value of $\alpha$ close to $166^{\circ}$, while no tip formation (i.e. $\alpha=$ $180^{\circ}$ ) is observed experimentally. In the case of $\mathrm{N}-\mathrm{BK} 7$, the initial value of $\gamma$ is significantly higher due to the fact that freezing begins at the droplet edge. The pointy tip begins to appear around $t=17 \mathrm{~s}$ when $\gamma$ has reached its minimum value of about $70^{\circ}$. The pointy tip formation is accompanied by a very sharp increase of $\gamma$. At the end of the freezing process, $\gamma$ is close to $100^{\circ}$ which is not far from $\gamma=90^{\circ}$ observed by (Marín et al. 2014).
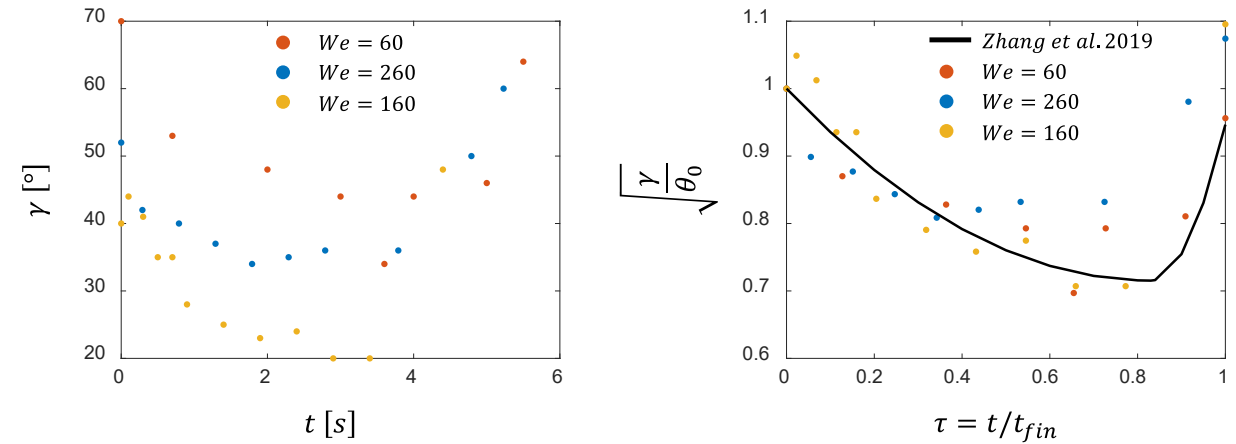

Figure 7 Evolution of the angle Igamma in function of the time $(\mathrm{A})$ and in function of the normalized time $\tau=t / t_{\text {fin }}$ (B) for different Weber number. Changing the Weber number allow us to change the angle $\theta_{0}$ made between the droplet and the substrate.

An accuracy of $\pm 5^{\circ}$ is expected in the current estimation of $\gamma$. With this end value of $\gamma$, a cone angle $\alpha$ of $125^{\circ}$ can be predicted by Eq.(13). This value is close to the measurement 
of $\alpha$ obtained from sideview images (about $120^{\circ} \pm 5^{\circ}$ ). More experiments undergone by changing the angle between the droplet and the surface tends to validate the model presented by (Zhang et al. 2019) (Figure 7)

\section{Conclusions}

A new experimental approach based on laser-induced fluorescence has been developed to reconstruct dynamically the ice front within a water droplet impinging on a subcooled solid substrate. This method benefits from the significant loss of fluorescence emission for certain fluorescent dyes when they are incorporated into an iced phase. As the fluorescence signal from the ice part is negligible, top view images of the deposited droplet can be safely considered for measuring the thickness of the liquid phase. The grey levels in the images closely correspond to an integration of the fluorescence signal over the thickness of the liquid layer. A second camera is necessary to provide side view images of the liquid/gas interface to account for the increase of the droplet volume during the freezing. This novel technique has made it possible to observe how the freezing dynamics influences the shape of the ice front. Experimentally it has been observed that the ice front takes a spherical shape. The study of two materials, namely duralumin and N-BK7 glass, indicates a very clear influence of the thermal properties of the solid substrate on the evolution of the ice front, most notably thermal diffusivity and effusivity. The ice front in the case of a N-BK7 substrate turns into a spherical cavity with a much deeper aspect than in the case of duralumin. This geometry is appropriate for the development of a pointy tip as the volume expands during the freezing.

\section{Nomenclature}

$\begin{array}{llll}c & \text { Concentration }\left[\mathrm{mol} . \mathrm{L}^{-1}\right] & V & \text { Volume }\left[\mathrm{m}^{3}\right] \\ C p & \text { Thermal heat conductivity }\left[\mathrm{J} . \mathrm{K}^{-1}\right] & Z & \text { Height }[\mathrm{m}] \\ D & \text { Thermal diffusivity }\left[\mathrm{m}^{2} \cdot \mathrm{s}^{-1}\right] & \alpha & \text { Cone angle }\left[{ }^{\circ}\right] \\ e & \text { Thermal effusivity }\left[\mathrm{J} \cdot \mathrm{K}^{-1} \cdot \mathrm{m}^{2} \mathrm{~s}^{-1 / 2}\right] & \rho & \text { Density }\left[\mathrm{kg} \cdot \mathrm{m}^{-3}\right] \\ \text { If } & \text { Intensity of fluorescence signal }\left[\mathrm{W} . \mathrm{m}^{2}\right] & \lambda & \text { Wavelength }[\mathrm{m}] \\ k & \text { Thermal conductivity }\left[\mathrm{W} \cdot \mathrm{m}^{-1} \cdot \mathrm{K}^{-1}\right] & V & \text { Thermal diffusivity ratio }[-] \\ L & \text { Latent heat }\left[\mathrm{J} \cdot \mathrm{kg}^{-1}\right] & \sigma & \text { Superficial tension }\left[\mathrm{N} . \mathrm{m}^{-1}\right] \\ R & \text { Radius }[\mathrm{m}] & V & \text { Angle at the tri-junction }\left[{ }^{\circ}\right] \\ t & \text { Time }[\mathrm{s}] & \Psi & \text { Density ratio }[-] \\ T & \text { Temperature }[\mathrm{K}] & I & \text { Ice } \\ u & \text { Velocity }\left[\mathrm{m} . \mathrm{s}^{-1}\right] & L & \text { Liquid }\end{array}$

\section{References}

Cebeci, T; Kafyeke F (2003) Aircraft icing. Annu Rev Fluid Mech 35:11-23

Hindmarsh JP, Wilson DI, Johns ML, House C (2005) NMR Verification of Single Droplet Freezing Models. 51:

Marín AG, Enríquez OR, Brunet $P$, et al (2014) Universality of tip singularity formation in freezing water drops. Phys Rev Lett 113:1-5

Schremb M, Roisman I V., Tropea C (2018) Normal impact of supercooled water drops onto a smooth ice surface: Experiments and modelling. J Fluid Mech 835:1087-1107

Schremb M, Webert T, Tropea C (2017) Experimental Investigation of Supercooled Water Drops Impacting onto a Smooth Ice Surface: Interaction of Fluid Flow and Phase Change. Proc 9th World Conf Exp Heat Transf Fluid Mech Thermodyn

Stiti M, Castanet G, Labergue A, Lemoine F (2020) Icing of a droplet deposited onto a subcooled surface. Int J Heat Mass Transf 159:120116

Thiévenaz V, Seón T, Josserand C (2019) Solidification dynamics of an impacted drop. J Fluid Mech 874:756-773

Zhang X, Liu X, Min J, Wu X (2019) Shape variation and unique tip formation of a sessile water droplet during freezing. Appl Therm Eng 147:927-934 